\title{
Identification of Differential Gene Expression Pattern in Lens Epithelial Cells Derived from Cataractous and Noncataractous Lenses of Shumiya Cataract Rat
}

\author{
Hidetoshi Ishida, ${ }^{1}$ Teppei Shibata, ${ }_{1}^{1}$ Yuka Nakamura, ${ }^{2}$ Yasuhito Ishigaki, ${ }^{2}$ \\ Dhirendra P. Singh, ${ }^{3}$ Hiroshi Sasaki, ${ }^{1}$ and Eri Kubo $\mathbb{1}^{1}$ \\ ${ }^{1}$ Department of Ophthalmology, Kanazawa Medical University, Ishikawa 9200293, Japan \\ ${ }^{2}$ Medical Research Institute, Kanazawa Medical University, Kanazawa, Ishikawa 9200293, Japan \\ ${ }^{3}$ Department of Ophthalmology, University of Nebraska Medical Center, Omaha, Nebraska 68198, USA \\ Correspondence should be addressed to Eri Kubo; kuboe@kanazawa-med.ac.jp
}

Received 25 June 2020; Revised 10 September 2020; Accepted 16 October 2020; Published 2 November 2020

Academic Editor: Marco Fichera

Copyright (c) 2020 Hidetoshi Ishida et al. This is an open access article distributed under the Creative Commons Attribution License, which permits unrestricted use, distribution, and reproduction in any medium, provided the original work is properly cited.

\begin{abstract}
The Shumiya cataract rat (SCR) is a model for hereditary cataract. Two-thirds of these rats develop lens opacity within 10-11 weeks. Onset of cataract is attributed to the synergetic effect of lanosterol synthase (Lss) and farnesyl-diphosphate farnesyltransferase 1 (Fdft1) mutant alleles that lead to cholesterol deficiency in the lenses, which in turn adversely affects lens biology including the growth and differentiation of lens epithelial cells (LECs). Nevertheless, the molecular events and changes in gene expression associated with the onset of lens opacity in SCR are poorly understood. In the present study, a microarray-based approach was employed to analyze comparative gene expression changes in LECs isolated from the precataractous and cataractous stages of lenses of 5-week-old SCRs. The changes in gene expression observed in microarray results in the LECs were further validated using real-time reverse transcribed quantitative PCR (RT-qPCR) in 5-, 8-, and 10-week-old SCRs. A mild posterior and cortical opacity was observed in 5-week-old rats. Expressions of approximately 100 genes, including the major intrinsic protein of the lens fiber (Mip and Aquaporin 0), deoxyribonuclease II beta (Dnase2B), heat shock protein B1 (HspB1), and crystallin $\gamma(\gamma C r y)$ $\mathrm{B}, \mathrm{C}$, and F, were found to be significantly downregulated (0.07-0.5-fold) in rat LECs derived from cataract lenses compared to that in noncataractous lenses (control). Thus, our study was aimed at identifying the gene expression patterns during cataract formation in SCRs, which may be responsible for cataractogenesis in SCR. We proposed that cataracts in SCR are associated with reduced expression of these lens genes that have been reported to be related with lens fiber differentiation. Our findings may have wider implications in understanding the effect of cholesterol deficiency and the role of cholesterol-lowering therapeutics on cataractogenesis.
\end{abstract}

\section{Introduction}

Age-related eye disease is a serious public health issue, and age-related cataract is the leading cause of blindness worldwide [1]. Currently, surgery is the only treatment for cataract. It has been reported that if the progression of cataract is delayed by 10 years, the huge expense associated with surgical intervention can be reduced [2]. Cataracts are caused by the degeneration of the lens protein called crystallin. Lenses have almost no protein turnover and are therefore suscepti- ble to ultraviolet rays, oxidative stress, and glycative stress. These stressors damage protein integrity and function leading to denaturation and aggregation of lens protein, which in turn results in lens opacification $[3,4]$. Recent studies have shown that oxidative stress controls various cellular processes associated with cell survival, such as cell proliferation, differentiation, aging, and cell death. It promotes cell apoptosis and senescence and is also associated with many diseases $[5,6]$. Oxidative stress and reactive oxygen species (ROS) are a major cause of age-related eye diseases, and diets rich in 
fruits, vegetables, vitamin C, zeaxanthin, lutein, and multivitamin-mineral supplements are recommended for preventing cataracts and age-related macular degeneration [7-10]. Moreover, several risk factors such as advancing age, genetic predisposition, oxidative stress, and external and internal factors acting adversely on lens homeostasis have been implicated to contribute to the etiology of cataract formation.

Cataracts are suggested to be a multifactorial disease associated with multiple etiological factors. To understand the molecular mechanism underlying cataract formation, animal models are a prerequisite. Shumiya cataract rats (SCRs) have been used as a model to study congenital cataract, exploring the initiation and development of the disease [7-9]. Lens opacity is observed in $66.7 \%$ of SCRs [10]. Onset of mature cataract in SCR occurs at around 10-11 weeks of age [10]. The vertebrate lens has a single layer of epithelial cells on its anterior surface. These cells are metabolic engines of lens and are responsible for maintaining its homeostasis and transparency. Any damage to lens epithelial cells (LECs) leads to cataractogenesis. Malfunction and aberrant differentiation of LECs contribute to cataractogenesis in SCRs [11]. Another interesting aspect is that the onset of cataract occurs in the lens epithelium in various types of cataract, such as selenite-induced cataract, UV-exposed cataract (in vitro), diabetic cataracts in rats, and noncongenital cataracts in humans [12-16]. The SCR is a model for hereditary cataract, but it also displays the features of oxidative stress-induced cataracts as its onset could be delayed by the administration of antioxidants [7]. Moreover, the genetic basis of cataractogenesis in SCRs is associated with the combination of lanosterol synthase (Lss) and farnesyl diphosphate farnesyl transferase 1 ( Fdft1) mutant alleles in the cholesterol biosynthesis pathway, lowering cholesterol levels in SCR lenses [16]. A previous study showed that inhibition of cholesterol synthesis inhibits cell proliferation in lens epithelial cells (LECs) [17], implicating that adequate cholesterol synthesis is required for maintaining the integrity and function of LECs, which in turn maintains normal lens biology and homeostasis. It has been found that mutations in Lss are one of the causes for the onset of human congenital cataracts $[18,19]$. Furthermore, lenses treated with lanosterol showed decreased opacity and increased transparency in the case of canine cataracts and reversed the aggregation of crystallin in vitro [18]. Hence, SCR is an appropriate model to study the mechanism underlying cataract formation and could help in the development of drugs for the treatment of cataracts.

In this study, we investigated the gene expression changes in LECs of SCRs to analyze the mechanism underlying cataract formation and its association with Lss mutation and cholesterol deficiency. A comprehensive analysis of gene expression changes in LECs from SCRs was analyzed using DNA microarray analysis to identify genes associated with cholesterol deficiency for the purpose of screening. Our study may identify the various genes contributing to the onset of congenital cataracts due to cholesterol deficiency. The outcomes of this study could contribute to the development of various therapeutic strategies to treat cataracts.

\section{Materials and Methods}

2.1. Animals. All animal experiments were approved by the Committee of Animal Research at Kanazawa Medical University (Permission no. 2017-107) and were conducted in accordance with the Guide for the Care and Use of Laboratory Animals implemented by the National Institutes of Health, the recommendations of the ARVO Statement for the Use of Animals in Ophthalmic and Vision Research, and the Institutional Guidelines for Laboratory Animals of Kanazawa Medical University. SCRs (SCR/Sscr: NBRP Rat No: 0823) were obtained from the National BioResource Project-Rat, Kyoto University (Kyoto, Japan).

We used 5-, 8- and 10-week-old SCRs in this study. Since mature cataracts develop in SCR after 11 weeks of age, rats with precataracts and mild to moderate cataracts were selected (5-10 weeks old). All rats were provided ad libitum access to regular or experimental chow (Sankyo Labo Service, Tokyo, Japan). The animals were sacrificed by administration of a lethal dose of $\mathrm{CO}_{2}$. Cataract onset in SCR was uniquely regulated by a specific combination of different mutant (Lss $\left.{ }^{1}\right)$ and polymorphic alleles $\left(L s s^{s}\right)$ on the Lss locus [16]. The prerequisite for cataract onset was the $L s s^{\mathrm{s}} / L s s^{1}$ genotype, which reduced LSS activity below the threshold that is about $12 \%$ of normal [16]. Lens opacity in SCR appears spontaneously at 9-11 weeks of age in 2/3 of animals $\left(L s s^{\mathrm{s}} / L s s^{1}\right)$. In $1 / 3$ of SCR $\left(L s s^{s} / L s s^{s}\right)$, no cataractous changes appeared even after 11 weeks of age. SCRs having Lss $/$ Lss 1 are embryonically lethal. Therefore, the rats were divided into two groups, i.e., control (Lss $\mathrm{s}^{\mathrm{s}} / \mathrm{Ls}^{\mathrm{s}}$; Cat-) and cataractous (Lss $\mathrm{s}^{\mathrm{s}} / \mathrm{Lss}^{\mathrm{l}}$; Cat+). Cat+ and Cat- SCRs were distinguished via PCR using genomic DNA isolated from the tails of 4-week-old rats. The amplified products were then separated on a $15 \%$ gel via polyacrylamide gel electrophoresis (PAGE) to detect Lss mutation $\left(L s s^{\mathrm{s}} / L s s^{\mathrm{s}}\right.$ or $\left.L s s^{\mathrm{s}} / L s s^{1}\right)$ as reported in a previous study of ours [20]. The sequences of primers used to detect Lss mutation were as follows: $5^{\prime}$-GCACACTGGACTGTGG CTGG- $3^{\prime}$ and $5^{\prime}$-GCCACAGCATTGTAGAGTCGCT- $3^{\prime}$.

2.2. RNA Extraction. Total RNA from each LEC sample obtained from SCR was extracted using the miRNeasy Micro Kit (Qiagen, Valencia, CA) following the manufacturer's protocol. Since RNA is extremely susceptible to degradation due to the ubiquitous presence of RNAses in the environment, purity and integrity of RNA were examined and validated as previously described [21]. Quality of total RNA was analyzed by evaluating the RNA integrity number (RIN) using Bioanalyzer RNA analysis (Agilent Technologies Japan Ltd., Tokyo, Japan). All RNA samples showed RIN > 9.0. In this study, we used LECs from SCR, because quality of total RNA obtained from whole lens in Cat+ SCR was poor showing RIN $<7.0$.

2.3. Microarray Analysis. Cataracts as well as Lss-mutationrelated genes were screened by microarray analysis using LEC samples from SCR as follows. Total RNAs from each LEC sample obtained from 2 eyes of 5-week-old Cat+ SCR with $L s s^{s} / L s s^{l}$ or Cat- SCRs with $L s s^{s} / L s s^{s}$ as the control were used for the microarray analysis $(n=1$, each). All samples 
were processed for the microarray analysis as follows: for RNA labeling and hybridization, GeneChip ${ }^{\mathrm{TM}}$ WT Pico Reagent Kit (Applied Biosystems, Thermo Fisher Scientific, Tokyo, Japan) and GeneChip ${ }^{\circledR}$ Rat gene 2.0 ST array (Affymetrix, Thermo Fisher Scientific) were used according to the manufacturer's protocol. Washing, scanning of the arrays, and analysis of scanned images were performed according to manufacturer's instructions. Each chip was normalized by dividing the measurement of each gene by the measurement of the specific control or by average intensity in the single array. Normalized data were exported for subsequent analysis. Genes with a normalized ratio $>2.0$-fold or $<0.5$-fold were selected as significant genes using the GeneSpring software package version 14.9 (Agilent). A Gene Ontology (GO) analysis was conducted using the DAVID database (https://david.ncifcrf.gov/) for the 110 downregulated genes in LECs of SCR Cat+.

2.4. Real-Time Reverse Transcriptase-Quantitative PCR (RT$q P C R)$. Total RNAs from each LEC sample obtained from 5-, 8-, and 10-week-old Cat+ SCR with $L^{s} s^{s} / L_{s}{ }^{l}$ and CatSCRs with $L s s^{s} / L s s^{s}$ as the control were used for the microarray analysis ( $n=3$, each). To measure the expression of rat deoxyribonuclease II beta (Dnase2B), heat shock protein B1 (HspB1), major intrinsic protein of lens (Mip), crystallin, gamma D $(\mathrm{Cry} \gamma \mathrm{D})$, heat shock transcription factor $4(H s f 4)$, lengsin $(L g s n)$, and tudor domain containing 7 ( $T d r d 7)$ mRNAs, we conducted a relative quantification of mRNA using Prism 7300 (Applied Biosystems, Thermo Fisher Scientific). The comparative $\mathrm{Ct}$ method was used for relative quantification of mRNA expression. The PCR amplification was performed with the TaqMan Universal PCR Master Mix. The probe mix containing the primers for Dnase2B, $H s p B 1, \gamma C r y$, and Mip was obtained from Thermo Fisher Scientific. All reactions were performed in triplicates. Differential expression for each gene was calculated using the comparative CT method using a predeveloped TaqMan Ribosomal RNA Control Reagent VIC probe as an endogenous control (Thermo Fisher Scientific).

2.5. Statistical Analysis. The statistical analysis was performed for all experiments using Student's $t$-test and/or one-way analysis of variance (ANOVA), as applicable. The data were presented as the mean \pm standard deviation (SD). A significant difference between the control and treatment group was defined as a $p$ value $<0.05$ for two or more independent experiments.

\section{Results}

3.1. Lens Morphology in 5- and 10-Week-Old SCRs. In CatSCR, the lens was clear, at 5 and 10 weeks of age, but in the case of Cat + SCR, a mild posterior and cortical opacity was observed at 5 weeks of age, and severe cortical and nuclear opacity was observed at age 10 weeks (Figure 1).

3.2. Analysis of Gene Expression Profile. As described in Materials and Methods, LECs from, 5-week-old Cat+ and Cat- SCR were used for microarray analysis $(n=1$ in each group) to screen genes associated with cholesterol deficiency

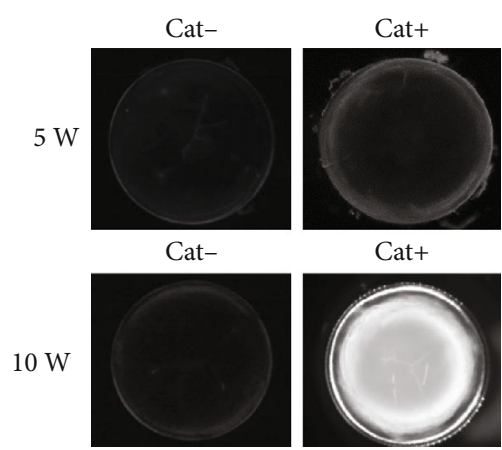

Figure 1: Observation of lens in 5- and 10-week-old Cat- and Cat+ SCRs. Lenses were extracted from 5- and 10-week-old SCRs and placed on glass bottom dishes containing Medium 199 (Thermo Fisher Scientific). Photographs of the lens were acquired and recorded with a stereo microscope.

and cataracts. The data for the microarray analysis was deposited to the Gene Expression Omnibus (GEO) database (Accession number: GSE152616). First, with 28,407 genes on the array, 110 genes were detected that showed a fold change $<0.5$ in the Cat+ group compared to those in Cat-, as shown in Figure 2. In the scatter plot, many genes were distributed in the lower part, and specifically, genes whose expression was downregulated could be detected using the scatter plot (Figure 2). Fold changes $(<0.5$-fold) were observed in 20 top-ranked genes (Table 1) in the microarray analysis including Dnase $2 B, H s p B 1, \gamma C r y$, Mip, beaded filament structural protein 1 ( $B f s p 1$ ), and Lgsn that have been reported to be associated with cataract and lens development [22-27]. Additionally, $H s f 4, T d r d 7$, gap junction protein epsilon 1 (Gje1), beaded filament structural protein 2 (Bfsp2), and lens intrinsic membrane protein $2(\operatorname{Lim} 2)$ which are also significantly reduced in the SCR lens $(<0.5$-fold) were linked to human or animal cataracts (Supplement 1) [27-33]. Furthermore, the expressions of six genes were upregulated (2.1- to 2.5-fold) in rat LECs from Cat+ compared to those from Cat-. Of the six genes, five genes were unidentified, and the other was Schlafen 4. However, the function of Schlafen 4 in the lens is not clear.

GO analysis revealed 110 downregulations of genes related to lens development, cellular water homeostasis, negative regulation of apoptotic process, etc. in LECs of Cat + SCR (Table 2).

3.3. Validation of Gene Expression Data Using RT-qPCR. The data from the microarray experiment shown above revealed the downregulation of various genes during the progression of cataracts. Therefore, we selected the following four genes: Dnase2B, Mip, HspB1, $\gamma$ Cry, Hsf4, Lgsn, and Tdrd7 that showed significant changes in expression according to the microarray data, and accordingly, we validated these results using RT-qPCR.

Data from the RT-qPCR showed that Dnase2B and Mip mRNA showed a significant downregulation in 5-, 8-, and 10-week-old Cat+ SCRs compared to Cat- SCRs (Figures 3(a) and 3(b)). The expressions of HspB1, Hsf4, Lgsn, and Tdrd7 mRNA significantly downregulated in 5- 
Fold change cut-off: 2.0111
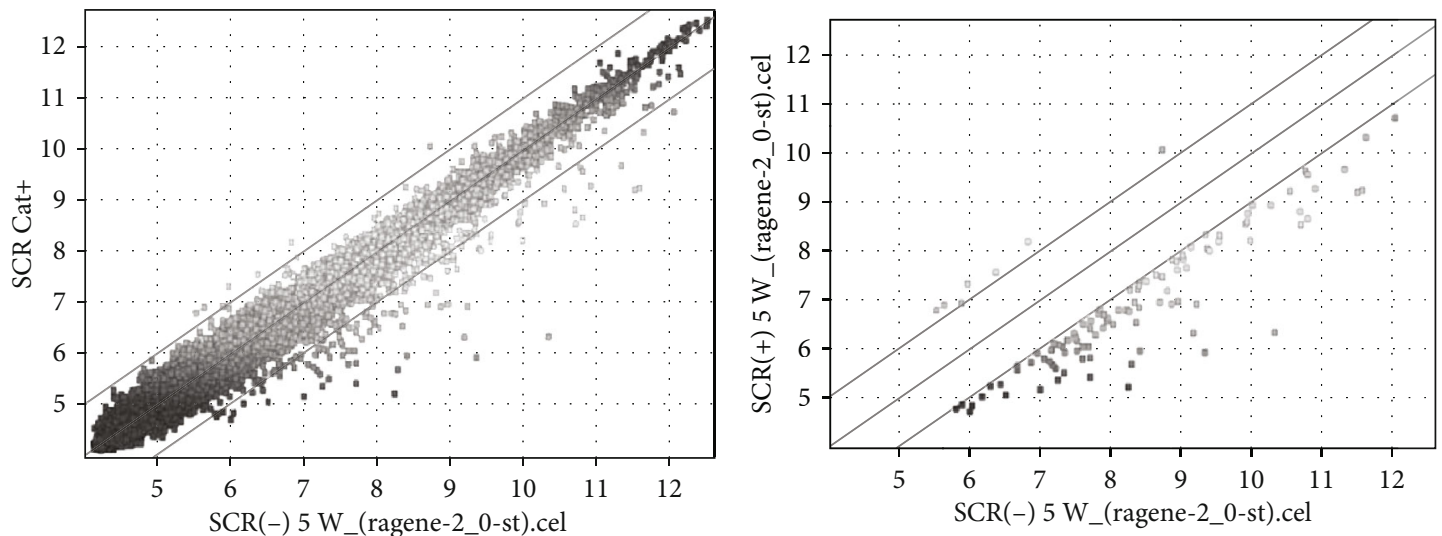

Figure 2: Analysis of gene expression profile. The scatter plots show a representative experiment using lens cDNAs from the Cat- and Cat+ SCRs at 5 weeks. Horizontal and vertical axes represent normal and experimentally generated signals on a logarithmic scale. The $x$-axis represents the $\log 2$ fold change, and the dark vertical lines represent cut-offs at 2 -fold decrease and increase.

TABLE 1: Lists of top 20 genes which are $<0.5$ downregulated in LECs in 5-week-old Cat+ SCR compared to Cat- SCR.

\begin{tabular}{|c|c|c|}
\hline Gene symbol & Gene description & FC \\
\hline Lgsn & Lens protein with glutamine synthetase domain & 0.064 \\
\hline Clic5 & Chloride intracellular channel 5 & 0.094 \\
\hline Snhg11 & Small nucleolar RNA host gene 11 & 0.124 \\
\hline Crygf & Crystallin, gammaF & 0.138 \\
\hline Srd5a2 & Steriod-5-alpha-reductase, alpha polypeptide 2 & 0.165 \\
\hline Cryge| Crygd & Crystallin, gamma E | crystallin, gamma D & 0.185 \\
\hline Mip & Major intrinsic protein of lens & 0.202 \\
\hline HspB1 & Heat shock protein $\mathrm{B} 1$ & 0.204 \\
\hline Crygc & Crystallin, gamma C & 0.206 \\
\hline Dnase2B & Deoxyribonuclease II beta & 0.207 \\
\hline Lctl & Lactase-like & 0.227 \\
\hline Bfsp1 & Beaded filament structural protein 1 , filensin & 0.231 \\
\hline Clic3 & Chloride intracellular channel 3 & 0.260 \\
\hline Fam89a & Family with sequence similarity 89 , member $7 \mathrm{~A}$ & 0.272 \\
\hline Cbln 2 & Cerebellin 2 precursor & 0.273 \\
\hline Mall & Mal, T-cell differentiation protein-like & 0.281 \\
\hline Wnt7a & Wingless-type MMTV integration site family, member 7A & 0.284 \\
\hline Slc24a2 & Solute carrier family 24 member 3 & 0.284 \\
\hline Hmox1 & Heme oxygenase 1 & 0.289 \\
\hline Lpin1 & Lipin1 & 0.302 \\
\hline
\end{tabular}

and 10-week-old Cat+ SCRs compared to Cat- SCRs (Figures 3(c), 3(e), 3(f), and 3(g)). However, the expression of Cry $\gamma D$ mRNA was significantly downregulated only in 5week-old Cat+ SCRs (Figure 3(d)).

\section{Discussion}

In this study, we comprehensively analyzed gene expression changes in the lens depending on the presence or absence of Lss deficiency in SCRs of different ages. SCRs with Lss deficiency gradually develop cataracts and show mature cataracts at 11 weeks of age. Lens opacity is slightly observed in 5- week-old SCRs with Lss mutations. In microarray analysis, 110 genes were downregulated with 0.5 -fold change in Cat+ SCR at 5 weeks of age. It is speculated that carrying the Lss mutation alters many gene expressions in the lens and induces lens opacity. In LECs of Cat+ SCRs with Lss mutations, the expressions of many genes reported to be associated with cataract development such as Dnase2B, HspB1, $\gamma C r y$, Mip, Hsf4, Tdrd7, Lim2, Gje1, Bfsp1, Bfsp2, and Lgsn were downregulated. In this study, we analyzed Dnase $2 B$, $H s p B 1, \gamma C r y$, and Mip genes using RT-qPCR. We confirmed that expressions of Dnase $2 B, H s p B 1$, and Mip mRNAs were significantly downregulated in the LECs of Cat+ SCRs with 
TABLE 2: GO analysis that showed changes of smaller than 0.5-fold in LECs from 5-week-old SCR Cat+ compared to SCR Cat-.

\begin{tabular}{lcc}
\hline GO accession & GO term & $p$ value \\
\hline GO:000208 & Lens development in camera-type eye & $6.18 E-06$ \\
GO:007030 & Lens fiber cell development & $5.05 E-04$ \\
GO:0034220 & Ion transmembrane transport & 0.003999 \\
GO:0007601 & Visual perception & 0.005318 \\
GO:0001654 & Eye development & 0.005798 \\
GO:0043010 & Camera-type eye development \\
GO:0008104 & Protein localization \\
GO:0015793 & Glycerol transport \\
GO:0009992 & Cellular water homeostasis \\
GO:0043627 & Response to estrogen \\
GO:0006833 & Water transport \\
GO:0009966 & Regulation of signal transduction & 0.021266 \\
GO:0043066 & Negative regulation of apoptotic process & 0.041241 \\
GO:0010976 & Positive regulation of neuron projection development & 0.044906 \\
GO:2001234 & Negative regulation of apoptotic signaling pathway & 0.051878 \\
\hline
\end{tabular}

Lss mutation before and after cataract onset. Furthermore, significant downregulation in the expression of $\gamma C r y$ mRNA was observed in the LECs of Cat+ SCRs with $L_{s s} / L s s^{l}$ only before the onset of the disease.

In this study, $H s f 4$ and $T d r d 7$ are the transcription factors (TF) that are found to be reduced in LECs of Cat+ SCR. It has been reported that $H s f 4$ mutation causes human congenital and age-related cataracts [28, 29]. Furthermore, the mutations of $T d r d 7$ also cause human congenital and age-related cataracts [30, 31]. Hsf4- knockout mice $\left(H s f 4^{-/-}\right)$and Tdrd7homozygous $\mathrm{KO}$ mice $\left(\mathrm{Tdrd} 7^{-1-}\right.$ ) also cause cataracts [32, 33]. Among 111 genes whose expression was decreased by LECs of Cat+ SCR, genes whose expression was also changed by the lens of Hsf4-conditional knockout mice (Hsf4-CKO) or $\mathrm{Td} d \mathrm{~d}$-homozygous $\mathrm{KO}$ mice $\left(\mathrm{Tdrd} \mathrm{T}^{/-}\right)$were analyzed using the iSyTE database (PMID: 29036527 and PMID: 32420594, respectively). Genes whose expressions were commonly reduced in LEC of Cat+ SCR and the lens of Hsf4-cKO were HspB1, Sh3bgr, Hmox1, Atp8a2, Pbld1, Bfsp1, Tdrd7, and Dnase $2 B$ (fold change $<0.5$ ) (Supplement 2). A gene whose expression was commonly reduced in LEC of Cat+ SCR and lenses of $T d r d 7^{1-}$ was HspB1 (fold change $<0.5$ ). Thus, HspB1 may induce the reduction of $H s f 4$ and/or Tdrd7 as TF in Cat+ SCR. Reduction of HspB1, Sh3bgr, Hmox1, Atp8a2, Pbld1, Bfsp1, and Dnase $2 B$ genes may be due to the downstream change by the $H s f 4$ regulation in Cat+ SCR.

HspB1 also known as heat shock protein 27 (Hsp27) is a protein that is encoded by the $H s p B 1$ gene in humans. HspB1 is an ATP-independent molecular chaperone with a conserved $\alpha \mathrm{B}$-crystalline domain in the $\mathrm{C}$-terminus region [34]. Heat shock proteins (Hsp) play a central role in maintaining cellular homeostasis and altering protein folding, thereby protecting $\alpha \mathrm{A}$ - and other crystalline proteins [35]. Further investigation of Hsp27 revealed that the protein responds to cellular oxidative and chemical stress conditions other than heat shock [35]. In the presence of oxidative stress, Hsp 27 plays a role as an antioxidant, decreasing the ROS by raising levels of intracellular glutathione (GSH) [36, 37]. It has been reported that mutation in $H s p B 1$ and/or $\alpha \mathrm{B}$-crystallin is responsible for the development of cataract [38] and is considered as major targets for the development of anticataract drugs [39]. We have previously reported that expression of Prdx6, an antioxidant protein, is decreased resulting in an increase in ROS in the LECs of Cat+ SCRs [7]. Thus, low activity of Lss may be involved in the decreased expression of antioxidant genes, causing oxidative stress and inducing cataracts in SCRs.

In mice, DNase II-like acid DNase (DLAD; Dnase $2 B)$ has been identified as a DNA-degrading enzyme that functions during lens enucleation [25]. Since the optimum $\mathrm{pH}$ for DLAD is acidic, it was suggested that the nucleus could be engulfed by lysosomes through autophagy and denucleated by the action of DLAD [25]. The lens consists of LECs and differentiated lens fiber cells. In the process of fiber differentiation, intracellular structures such as the nucleus, mitochondria, and endoplasmic reticulum disappear and the lens becomes transparent. In the process of enucleation, the DNA that encodes the genetic information is degraded. For this enucleation process, DLAD plays an important role. In Dlad knockout mice, the eye lens seemed to have developed normally; however, undegraded DNA was observed in the lens fiber that contributed to lens opacity. Thus, DLAD is necessary to maintain lens transparency and normal fiber differentiation. Lss mutation downregulates the expression of Dlad (Dnase2B), which could be attributed to cataract development in SCRs.

MIP is a lens fiber major intrinsic protein, also known as Aquaporin 0, which is a water channel in lens fiber cells, facilitating the movement of water, gap junction channels, and solute transporters [40]. MIP plays a crucial role in regulating the osmolarity and homeostasis of the lens and stabilizing cell junctions in the lens nucleus. Currently, 12 mutations in MIP have been linked to autosomal-dominant cataracts in humans [26]. The decreased expression of Mip in LEC of 


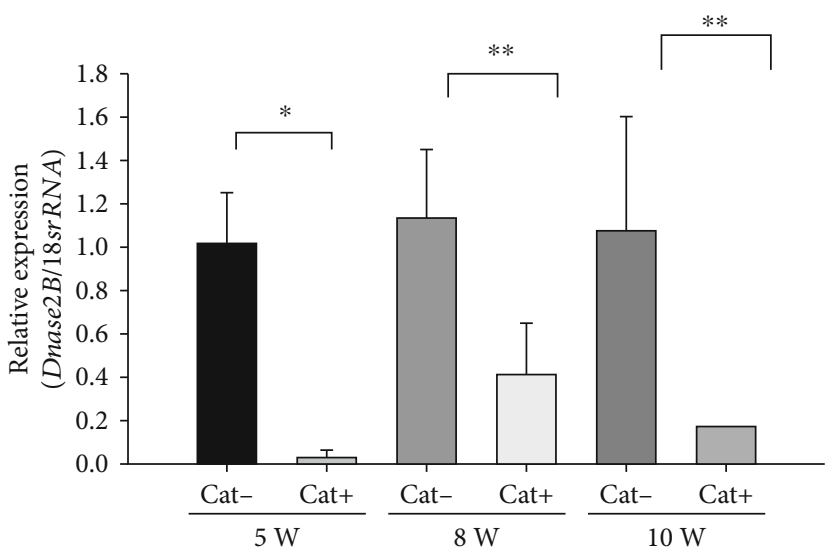

(a)

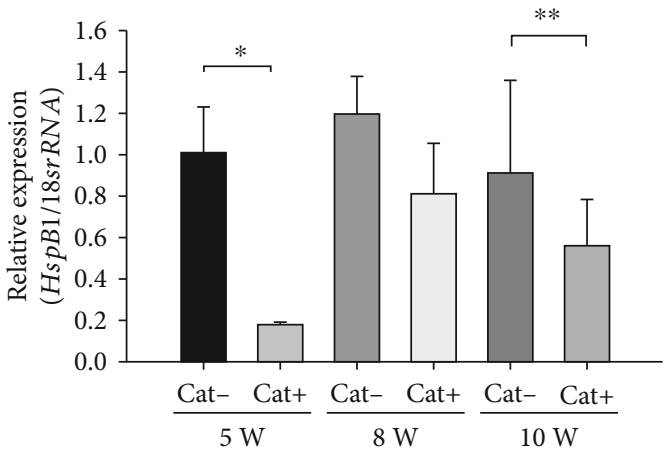

(c)

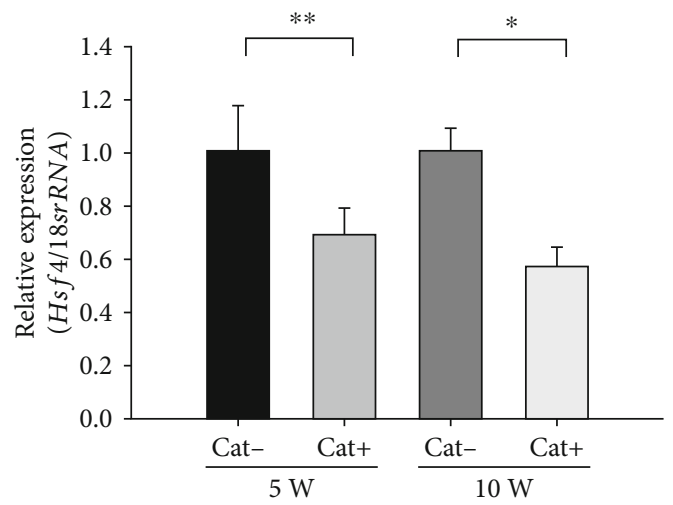

(e)

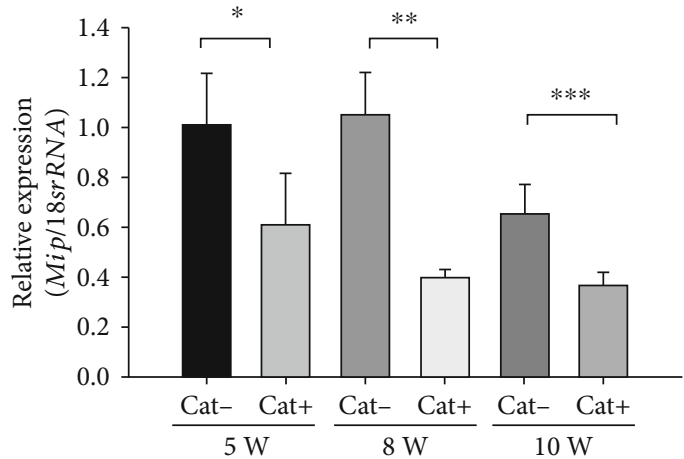

(b)

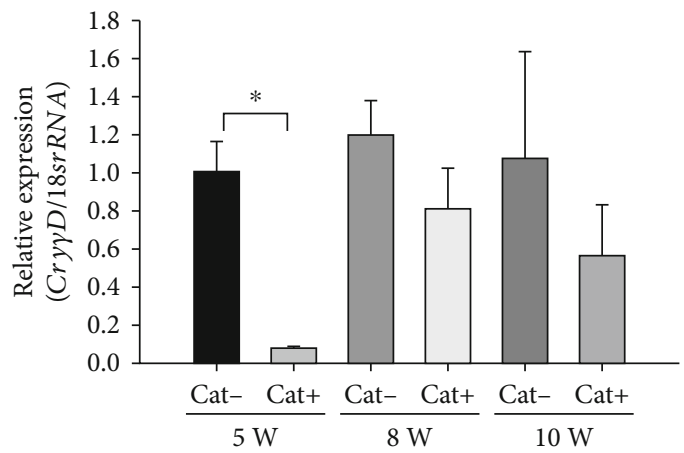

(d)

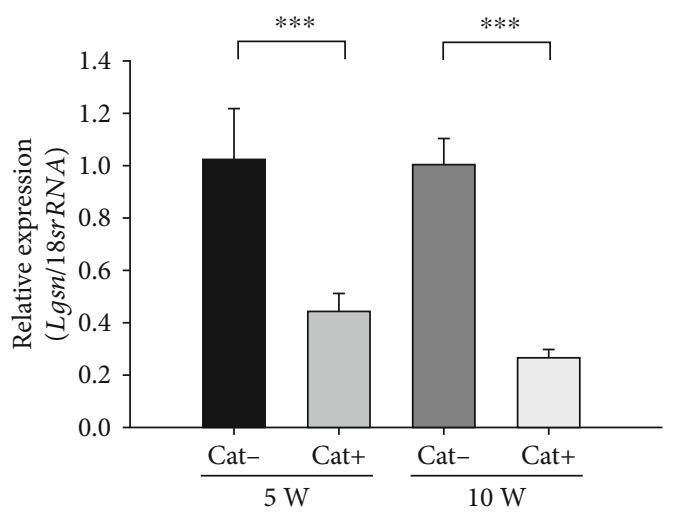

(f)

Figure 3: Continued. 


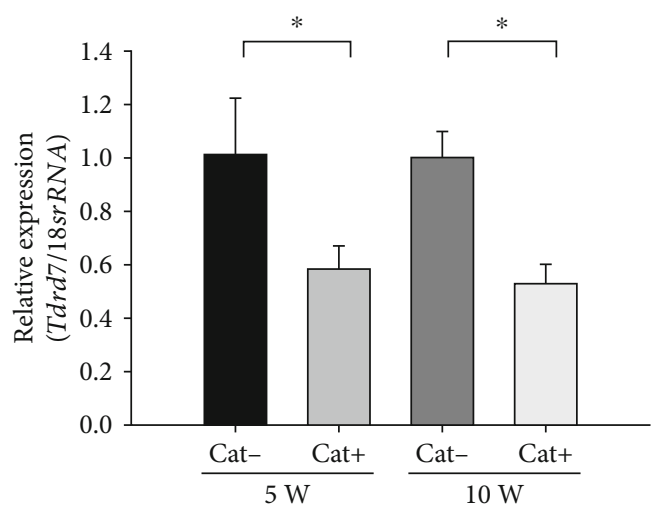

(g)

Figure 3: Expressions of Dnase2B, Mip, HspB1, Cry $\gamma D, H s f 4, L g s n$, and Tdrd7 mRNA in LECs from Cat- SCR and Cat+ SCR at 5, 8, and 10 weeks of age or 5 and 10 weeks of age. (a) Expression of Dnase $2 B$ mRNA: ${ }^{*} p<0.02,{ }^{* *} p<0.04$. (b) Expression of Mip mRNA: ${ }^{*} p<0.02$, ${ }^{* * *} p<0.001,{ }^{* *} p<0.04$. (c) Expression of HspB1 mRNA: ${ }^{*} p<0.02,{ }^{* *} p<0.04$. (d) Expression of Cry $\gamma D$ mRNA: ${ }^{*} p<0.02,{ }^{* * *} p<0.001$, ${ }^{* *} p<0.04$. (e) Expression of Hsf4 mRNA: ${ }^{*} p<0.02,{ }^{* *} p<0.04$. (f) Expression of Lgsn mRNA: ${ }^{* * *} p<0.001$. (g) expression of Tdrd7 mRNA: ${ }^{*} p<0.02$. Data are expressed as the mean \pm standard deviation $(n=3)$.

Cat+ SCRs may disrupt cellular water homeostasis and induce lens fiber swelling and vacuole formation observed in SCR lenses.

The nuclear region of the eye lens is particularly rich in the $\gamma$ Cry protein, which is necessary to maintain structural and functional properties in the lens. $\gamma$ Cry mRNA was downregulated in the LECs of Cat+ SCRs before the onset of lens opacity compared to Cat- rats. However, there was no significant change in expression of this gene after the onset of cataracts. It is not clear why $\gamma$ Cry mRNA expression did not decrease in cataractous lenses in SCRs. Further studies are required to understand this phenomenon.

Sterol, such as cholesterol in animals, is a compound that is important as a biosynthetic raw material for steroid hormones, vitamin $\mathrm{D}$, and bile acids, in addition to its role in regulating membrane fluidity as a component of eukaryotic biological membranes [41]. Cholesterol synthesis is carried out through a process of approximately 30 enzymatic reactions using acetyl-CoA as a starting substrate. Lanosterol is the first sterol in the cholesterol synthesis pathway and LSS is an essential rate-limiting enzyme that functions as a downstream element in the lanosterol biosynthetic pathway, catalysing the cyclization of the linear 2,3-monoepoxysqualene to cyclic lanosterol [42]. Congenital cataracts with homozygous and heterozygous Lss mutations have been reported to affect the catalysing functions of Lss [18, 19]. Furthermore, the polymorphism rs2968 of the Lss gene was associated with nuclear type of age-related cataract (ARC) risk in the Chinese population [43]. Consequently, it has been reported that the mRNA expression of Lss was significantly lower in LECs of all subtypes of the ARC group than the control group [43]. Epidemiological studies have shown that individuals receiving statins, which are cholesterol synthesis inhibitors, have an increased risk of being diagnosed with cataracts [44, 45]. Previous studies have reported that Lss might play a significant role in oxidative stress and maintenance of lens transparency [46]. These results indicate that Lss deficiency may be a risk factor of ARC. Additionally, it has also been reported that lanosterol plays a preventive role in cataract formation, inhibiting lens opacity and reversing crystalline aggregation [18]. Additionally, intravitreal injection of lanosterol nanoparticles has been reported to rescue the early stage of lens damage in SCRs [8]. Thus, synthesis of cholesterol by LSS is important to maintain lens transparency.

\section{Conclusions}

In conclusion, our study demonstrated that Lss mutations in SCRs result in reduction of $\mathrm{Hsf} 4$ and Tdrd7 inducing the downregulation of several genes associated with maintaining lens transparency and identified their relationship with cholesterol deficiency. Cholesterol and Lss in lenses may be important to maintain normal lens homeostasis such as lens fiber differentiation, oxidative and heat stresses, and regulation of lens osmolarity to maintain lens transparency.

\section{Data Availability}

All data generated or analyzed during this study are included in this published article. More details are available from the corresponding author upon reasonable request.

\section{Conflicts of Interest}

There was no conflict of interest.

\section{Acknowledgments}

We are thankful to the National BioResource Project-Rat (http://www.anim.med.kyoto-u.ac.jp/NBR/) for providing the rat strains (SCR/Sscr: NBRP Rat No. 0823). We would like to thank Editage (http://www.editage.com) for English language editing. This work was supported by grants from the Japan Society for the Promotion of Science (JSPS KAKENHI, Grant Number JP 17K11470 to EK) and National Eye Institute, National Institute of Health (NIH) (EY024589) to (DPS). 


\section{References}

[1] R. R. A. Bourne, G. A. Stevens, R. A. White et al., "Causes of vision loss worldwide, 1990-2010: a systematic analysis," The Lancet Global Health, vol. 1, no. 6, pp. e339-e349, 2013.

[2] G. Brian and H. Taylor, "Cataract blindness-challenges for the 21st century," Bulletin of the World Health Organization, vol. 79, no. 3, pp. 249-256, 2001.

[3] J. R. Chang, E. Koo, E. Agrón et al., "Risk factors associated with incident cataracts and cataract surgery in the Agerelated Eye Disease Study (AREDS): AREDS report number 32," Ophthalmology, vol. 118, no. 11, pp. 2113-2119, 2011.

[4] C. A. McCarty, M. B. Nanjan, and H. R. Taylor, "Attributable risk estimates for cataract to prioritize medical and public health action," Investigative Ophthalmology \& Visual Science, vol. 41, no. 12, pp. 3720-3725, 2000.

[5] B. N. Ames, M. K. Shigenaga, and T. M. Hagen, "Oxidants, antioxidants, and the degenerative diseases of aging," Proceedings of the National Academy of Sciences of the United States of America, vol. 90, no. 17, pp. 7915-7922, 1993.

[6] C. Lopez-Otin, M. A. Blasco, L. Partridge, M. Serrano, and G. Kroemer, "The hallmarks of aging," Cell, vol. 153, no. 6, pp. 1194-1217, 2013.

[7] E. Kubo, N. Fatma, Y. Akagi, D. R. Beier, S. P. Singh, and D. P. Singh, "TAT-mediated PRDX6 protein transduction protects against eye lens epithelial cell death and delays lens opacity," American Journal of Physiology-Cell Physiology, vol. 294, no. 3, pp. C842-C855, 2008.

[8] N. Nagai, Y. Fukuoka, K. Sato et al., "The intravitreal injection of lanosterol nanoparticles rescues lens structure collapse at an early stage in Shumiya cataract rats," International Journal of Molecular Sciences, vol. 21, no. 3, article 1048, 2020.

[9] M. Oka, H. Kudo, N. Sugama, Y. Asami, and M. Takehana, "The function of filensin and phakinin in lens transparency," Molecular Vision, vol. 14, pp. 815-822, 2008.

[10] S. Shumiya, "Establishment of the hereditary cataract rat strain (SCR) and genetic analysis," Laboratory Animal Science, vol. 45, no. 6, pp. 671-673, 1995.

[11] T. Okano, S. Uga, S. Ishikawa, and S. Shumiya, "Histopathological study of hereditary cataractous lenses in SCR strain rat," Experimental Eye Research, vol. 57, no. 5, pp. 567-576, 1993.

[12] K. R. Hightower and J. P. McCready, "Effect of selenite on epithelium of cultured rabbit lens," Investigative Ophthalmology \& Visual Science, vol. 32, no. 2, pp. 406-409, 1991.

[13] W. C. Li and A. Spector, "Lens epithelial cell apoptosis is an early event in the development of UVB-induced cataract," Free Radical Biology \& Medicine, vol. 20, no. 3, pp. 301-311, 1996.

[14] P. F. Kador and J. H. Kinoshita, "Diabetic and galactosaemic cataracts," Ciba Foundation Symposium, vol. 106, pp. 110131, 1984.

[15] W. C. Li, J. R. Kuszak, K. Dunn et al., "Lens epithelial cell apoptosis appears to be a common cellular basis for noncongenital cataract development in humans and animals," The Journal of Cell Biology, vol. 130, no. 1, pp. 169-181, 1995.

[16] M. Mori, G. Li, I. Abe et al., "Lanosterol synthase mutations cause cholesterol deficiency-associated cataracts in the Shumiya cataract rat," The Journal of Clinical Investigation, vol. 116, no. 2, pp. 395-404, 2006.

[17] G. N. el-Sayed and R. J. Cenedella, "Relationship of cholesterolgenesis to DNA synthesis and proliferation by lens epithelial cells in culture," Experimental Eye Research, vol. 45, no. 3, pp. 443-451, 1987.

[18] L. Zhao, X. J. Chen, J. Zhu et al., "Lanosterol reverses protein aggregation in cataracts," Nature, vol. 523, no. 7562, pp. 607611, 2015.

[19] X. Chen and L. Liu, "Congenital cataract with LSS gene mutations: a new case report," Journal of Pediatric Endocrinology and Metabolism, vol. 30, no. 11, pp. 1231-1235, 2017.

[20] H. Ishida, T. Shibata, S. Shibata, Y. Tanaka, H. Sasaki, and E. Kubo, "Lutein plus water chestnut (Trapa bispinosaRoxb.) extract inhibits the development of cataracts and induces antioxidant gene expression in lens epithelial cells," BioMed Research International, vol. 2020, Article ID 9204620, 9 pages, 2020.

[21] T. Maniatis, E. F. Fritsch, and J. Sambrook, Molecular Cloning. A Laboratory Manual, Cold Spring Harbor Laboratory, 1982.

[22] U. P. Andley, “ $\alpha \mathrm{A}$-crystallin $\mathrm{R} 49 \mathrm{C}^{\text {neo }}$ mutation influences the architecture of lens fiber cell membranes and causes posterior and nuclear cataracts in mice," BMC Ophthalmology, vol. 9, no. 1, p. 4, 2009.

[23] F. Grassi, N. Moretto, C. Rivetti et al., "Structural and functional properties of lengsin, a pseudo-glutamine synthetase in the transparent human lens," Biochemical and Biophysical Research Communications, vol. 350, no. 2, pp. 424-429, 2006.

[24] M. Kumar, T. Agarwal, S. Khokhar et al., "Mutation screening and genotype phenotype correlation of alpha-crystallin, gamma-crystallin and GJA8 gene in congenital cataract," Molecular Vision, vol. 17, pp. 693-707, 2011.

[25] S. Nishimoto, K. Kawane, R. Watanabe-Fukunaga et al., "Nuclear cataract caused by a lack of DNA degradation in the mouse eye lens," Nature, vol. 424, no. 6952, pp. 10711074, 2003.

[26] X. Shentu, Q. Miao, X. Tang, H. Yin, and Y. Zhao, "Identification and functional analysis of a novel MIP gene mutation associated with congenital cataract in a Chinese family," PLoS One, vol. 10, no. 5, article $\mathrm{e} 0126679,2015$.

[27] H. Wang, T. Zhang, D. Wu, and J. Zhang, "A novel beaded filament structural protein 1 (BFSP1) gene mutation associated with autosomal dominant congenital cataract in a Chinese family," Molecular Vision, vol. 19, pp. 2590-2595, 2013.

[28] Y. Shi, X. Shi, Y. Jin et al., "Mutation screening of HSF4 in 150 age-related cataract patients," Molecular Vision, vol. 14, pp. 1850-1855, 2008.

[29] N. Smaoui, O. Beltaief, S. BenHamed et al., "A homozygous splice mutation in theHSF4Gene is associated with an autosomal recessive congenital cataract," Investigative Ophthalmology \& Visual Science, vol. 45, no. 8, pp. 2716-2721, 2004.

[30] D. W. Shao, C. Y. Yang, B. Liu et al., "Bioinformatics analysis of potential candidates for therapy of TDRD7 deficiencyinduced congenital cataract," Ophthalmic Research, vol. 54, no. 1, pp. 10-17, 2015.

[31] C. Zheng, M. Wu, C. Y. He et al., "RNA granule component TDRD7 gene polymorphisms in a Han Chinese population with age-related cataract," The Journal of International Medical Research, vol. 42, no. 1, pp. 153-163, 2014.

[32] M. Fujimoto, H. Izu, K. Seki et al., "HSF4 is required for normal cell growth and differentiation during mouse lens development," The EMBO Journal, vol. 23, no. 21, pp. 4297-4306, 2004.

[33] C. E. Barnum, S. Al Saai, S. D. Patel et al., "The Tudor-domain protein TDRD7, mutated in congenital cataract, controls the 
heat shock protein HSPB1 (HSP27) and lens fiber cell morphology," Human Molecular Genetics, vol. 29, no. 12, pp. 2076-2097, 2020.

[34] B. Lelj-Garolla and A. G. Mauk, "Roles of the N- and Cterminal sequences in Hsp27 self-association and chaperone activity," Protein Science, vol. 21, no. 1, pp. 122-133, 2012.

[35] L. Urbak and H. Vorum, "Heat shock proteins in the human eye," International Journal of Proteomics, vol. 2010, Article ID 479571, 8 pages, 2010.

[36] P. Mehlen, E. Hickey, L. A. Weber, and A. P. Arrigo, "Large unphosphorylated aggregates as the active form of hsp27 which controls intracellular reactive oxygen species and glutathione levels and generates a protection against TNFalpha in NIH-3T3-ras cells," Biochemical and Biophysical Research Communications, vol. 241, no. 1, pp. 187-192, 1997.

[37] A. P. Arrigo, S. Virot, S. Chaufour, W. Firdaus, C. Kretz-Remy, and C. Diaz-Latoud, "Hsp27 consolidates intracellular redox homeostasis by upholding glutathione in its reduced form and by decreasing iron intracellular levels," Antioxidants \& Redox Signaling, vol. 7, no. 3-4, pp. 414-422, 2005.

[38] U. P. Andley, E. Tycksen, B. N. McGlasson-Naumann, and P. D. Hamilton, "Probing the changes in gene expression due to $\alpha$-crystallin mutations in mouse models of hereditary human cataract," PLoS One, vol. 13, no. 1, article e0190817, 2018.

[39] A. P. Arrigo, S. Simon, B. Gibert et al., "Hsp27 (HspB1) and alphaB-crystallin (HspB5) as therapeutic targets," FEBS Letters, vol. 581, no. 19, pp. 3665-3674, 2007.

[40] S. Bassnett, P. A. Wilmarth, and L. L. David, "The membrane proteome of the mouse lens fiber cell," Molecular Vision, vol. 15, pp. 2448-2463, 2009.

[41] O. Foresti, A. Ruggiano, H. K. Hannibal-Bach, C. S. Ejsing, and P. Carvalho, "Sterol homeostasis requires regulated degradation of squalene monooxygenase by the ubiquitin ligase Doa10/Teb4," eLife, vol. 2, article e00953, 2013.

[42] M. W. Huff and D. E. Telford, "Lord of the rings-the mechanism for oxidosqualene:lanosterol cyclase becomes crystal clear," Trends in Pharmacological Sciences, vol. 26, no. 7, pp. 335-340, 2005.

[43] X. Zou, H. Wang, D. Zhou et al., "The polymorphism rs2968 of LSS Gene confers susceptibility to age-related cataract," DNA and Cell Biology, 2020.

[44] S. N. Al-Holou, W. R. Tucker, E. Agrón et al., "The association of statin use with cataract progression and cataract surgery: the AREDS2 report number 8," Ophthalmology, vol. 123, no. 4, pp. 916-917, 2016.

[45] J. C. Erie, M. R. Pueringer, S. M. Brue, A. M. Chamberlain, and D. O. Hodge, "Statin use and incident cataract surgery: a casecontrol study," Ophthalmic Epidemiology, vol. 23, no. 1, pp. 40-45, 2016.

[46] H. Hua, T. Yang, L. Huang et al., "Protective effects of lanosterol synthase up-regulation in UV-B-induced oxidative stress," Frontiers in Pharmacology, vol. 10, p. 947, 2019. 